Volume. 5 Number. 1

Period: January - June 2021; page 9-16

Jurnal Ilmiah Keperawatan Orthopedi

p-ISSN : 2580-1112; e-ISSN : 2655-6669

(JIKO)

Copyrighr@2020

Article history:

The author owns the copyright of this article

journal homepage: https://ejournal.akperfatmawati.ac.id

DOI : $10.46749 /$ jiko.v5i1.56

Received: February 23, 2021

Revised: February 28, 2021

Accepted: March 03, 2021

\title{
Isometric Exercise of Quadriceps and Gluteal Muscle in Patient with Close Femur Fracture
}

\author{
Sri Wirayuni ${ }^{1}$, Liya Arista ${ }^{2}$ \\ ${ }^{1}$ Faculty of Nursing, University of Indonesia, Depok - Indonesia \\ E-mail:_sriwirayuni07@gmail.com
}

\begin{abstract}
Femur fracture is a fracture in the largest bone in the body. The femur requires significant force to fracture. One of the treatments for femoral fracture is reduction and immobilization using traction. Immobilization in traction-attached patients before preoperatively requires isometric exercises to maintain muscle strength. Isometric exercise is a muscle contraction exercise, the patient contracts the muscles without moving the muscles to maintain muscle and prevent atrophy. The purpose of this paper is to identify the effectiveness of isometric exercises to increase isometric muscle strength in patients with lower limb fracture. The methodology used is a case study. The application of isometric exercises can increase muscle strength in patients with skin traction attached. Nurses need to pay attention to factors that can affect the patient's ability to perform isometric exercises so that the results obtained can be more optimal and the patient experiences an increase in the ability of his limbs.
\end{abstract}

Keywords : Isometric Exercises; Muscle Strength, lower limb fracture

\section{Introduction}

The femur is part of the lower limb where the lower limb has the highest injury problems, which is about $67 \%$ and causes scars that interfere with comfort around $9.2 \%$. The condition of the house and its environment has the highest potential to cause injury to this fracture, which is around $44.7 \%$ compared to the incidence of cid era due to traffic around $31.4 \%$ (Riskesdas, 2018)

Femur fracture is a fracture in the largest bone in the body. The femur requires significant force to fracture. In the elderly, the mechanism with low energy can break the bone, resulting in the spiral conjunction (Willmott, 2015). Fracture of femoral stem is a break of continuity that can result from direct trauma (traffic accidents, falls from heights), and usually more experienced by adult men. A fracture in this area can cause significant bleeding, and can cause sufferers to experience it (LeMone, Burke, \& Bauldoff, 2016).

Cases of femoral fracture is the most common which is approximately $39 \%$, followed by $15 \%$ of the humerus fractures, fractures of the tibia and fibula $11 \%$, of which the biggest cause of fracture of the femur is an accident of traffic that usually disebabk's by accident mobil, motorcycle or vehicle $62.6 \%$ and $\mathrm{n}$ fell from a height of $37.3 \%$. The incidence of femoral fractures in women is the second most common fracture $(17.0 \%$ of 10,000 people / year) and number seven in men (5.3\% of 10,000 people / year). The peak age distribution in femoral fractures is adults (15-34 years) and the elderly (over 70 years) (Amponsah \& Gorleku, 2015).

Treatment for patients with femur consists of closed reduction and the reduction of open through operations such as immobilization with a 
spica cast, fixation of external and internal fixation, nailing intermedularry with te $\mathrm{kn} \mathrm{ik} \mathrm{terbu} \mathrm{ka} \mathrm{or}$ closed nailing intermedularry antegrade interlocked with or without reaming, nailing retrograde interlock intramedularry and plate fixation ( $\mathrm{Ru}$ dloff, 2020). The type and location of the fracture, degree of comminution, patient age, socioeconomic status and other factors can influence the method of fracture management.

Agreed principles in the management of facial fractures include: (1) restoration of alignment, rotation and length of the fracture, (2) maintenance of the blood supply to help maintain and prevent infection, and (3) rehabilitation of the limb and patient. Actions performed on patients with femoral fractures depend on the type of fracture selected from the $\mathrm{x}$ ray results, namely traction, ORIF plate and screw, intermedulary nailing (Willmott, 2015).

Traction is giving tensile force to the body part, is used to minimize muscle spasm, mer e duction, align and clicking imobilisasikan fracture, reduce deformity and to increase the space between the two fracture. The type of traction used depends on the goal to be achieved, namely skin traction to control spasm and immobilization as well as secondary traction to immobilize the fracture.

Black ( 2014 ) explains that the type of skin traction is used for cases of the lower leg, lower back, upper leg of the arm, while bone traction is used in supracondillary femur fractures (some are continuous or dynamic). BUCK skin traction is used for immobilization before surgery in femur fracture patients.

Immediately after the installation of traction, the patient feels weak and unstable as a result of muscle atrophy or hypersiortostatics, so it is not necessary to pay attention to the intervention that will be given because it can have further impacts. Deep

vein thrombosis (DVT), pressure sores, congestive fever and pneumonia, constipation, anorexia, and urinary infections are some of the effects of traction.

DVT or trombos is a vein in a venous thrombolysis in and caused by patients who have a lifespan of over 40 years who runs a great operation, orthopedic or cardiac infarction. Under normal conditions, venous return will be assisted by the pumping of the calf muscles, on the other hand, when the legs are not moving, the pumping is not effective, there will be blood dams due to venous gravity.

Tombus formation is an initial local process that starts from the formation of platelets in the endothelium wall. The most dangerous complication of DVT is the occurrence of pulmonary embolism. The embolus that moves to the lung causes a decrease in the perfusion of the part of the lung that is supplied by blood and an imbalance in the lung will occur and results in hypoxemia. Embolus that occurs in large vessels in the lung will increase proximal pulmonary vascular resistance and result in ateletaksis and ultimately decrease cardiac output (right heart failure).

Anticoagulant therapy is given to patients with DVT while the prevention of DVT can be done with muscle training and ambulation (Black, JM, \& Hawks, 2014).

Muscle training is very useful for maintaining muscle strength and tone and improving blood circulation. Muscle exercises are adapted to the therapeutic limitations of traction, whereas active muscle exercises include pulling the grip on the bed, leg flexion and tension, range of motion and weight bearing exercises for healthy joints.

In immobilized extremities, isometric exercises, quadriceps exercises and glute al adjustments are very important to maintain the strength of the large muscles that 
function in walking. Isometric exercise is a muscle contraction exercise, the patient is trained to tighten the muscles without moving the muscles to maintain muscle mass and prevent arophyria. Quardricep and gluteal exercises are exercises to tense muscles to keep the muscles awake. Based on the description above, the authors are interested in understanding the benefits of isometric, quadricep and gluteal muscle strength training in patients with segmental close fracture 1/3 distal left femur in the Angsoka 3 treatment room, Sanglah Hospital Denpasar, Bali .

\section{Method}

This study used case studies method of real case in hospital and then looking for theoretical references that are relevant to the cases or problems found. Literature study is a method used to collect data or sources that are related to the topic being raised.

Literature studies are obtained from various sources, namely journals, nursing documentation books and literature (Surahman \& Rachmat, 2016)

\section{Case Description}

Mrs. M, as a housewife with the age of 45 came to the emergency room of the Sanglah General Hospital on September, 212020 at 9:00 a.m. the patient came with complaints of pain in her left leg after the patient was previously taken to the Hospital, escorted by families and officers using the brancard. The patient presented with a spalk on the left leg and was attached to an infusion of RL 28 drops per minute.

Patients previously experienced a traffic accident traffic, when riding a motorcycle, the patient is hit from the left by using sepe da motors. After getting treatment in ER patients paired skin traction with a weight of 5 kilograms. Patient get paracetamol every 6 hours, and cefixime $100 \mathrm{mg}$ every 12 hours. The medical diagnosis is Close segmental fractures of the distal femur middle $1 / 3$ of the left and the planned for surgery and installation of intermedularry nailing on theleft leg.

Patient has pain in the arms and left leg. Pain assessment consist of, P: activity and movement of extremity, Q: intermittent on their right hand and left foot, R: on left hand and foot, $S$ : pain scale 3, T: pain duration for 15 minutes continuously. The patient also experienced sensory changes. The patient felt pain without a tingling sensation in the hand that was attached to the cast. The patient also complained of swelling in the patient's left hand and leg at the start of the fall but at this time the swelling was reduced. The swelling in the left hand decreased after placing the cast.

Meanwhile, the deformity in the leg was no longer visible, because the patient had a cast and traction attached. In the examination results of physical general ti dak can be done for the patient in a position that can not be moved, while the results of the examination showed:

Look (Inspection) foot show visible swelling and hematoma, but no deformity because the foot is attached skin traction weighing 5 kilograms.

Traction is mounted on the patient is still in good condition (no loose straps, the load does not touch the floor, no redness on the skin attached traction ). On palpation, the Feel examina tion was performed, there was tenderness in the thigh area but did not increase, there was no muscle spasm. Crepitations bone of movement in the area frak tours can be touched, but when pengka jian s 've not studied again for at leg fracture.

Assessment of Movement is not done at all joints in the left leg as the patient is already installed skin traction. The examinations were carried out on the distal area of the left foot, namely dorsiflexion and plant ar flexion 
of the left ankle, inversion and eversion and movement of the toes. Laboratory examination results: $\mathrm{Hb}$ : 9.05 gr/DL, Hct $28.21 \%$, Aptt 20.27 seconds, INR 16, SGOT $4.3 \mathrm{U} /$ L.

Meanwhile, the X-ray of the femur showed that the patient had a segmental fracture in the left middle femur distal, diplacement $(+)$, shortening $(+)$, with tissue swelling around emphysema subutis genu genu left.

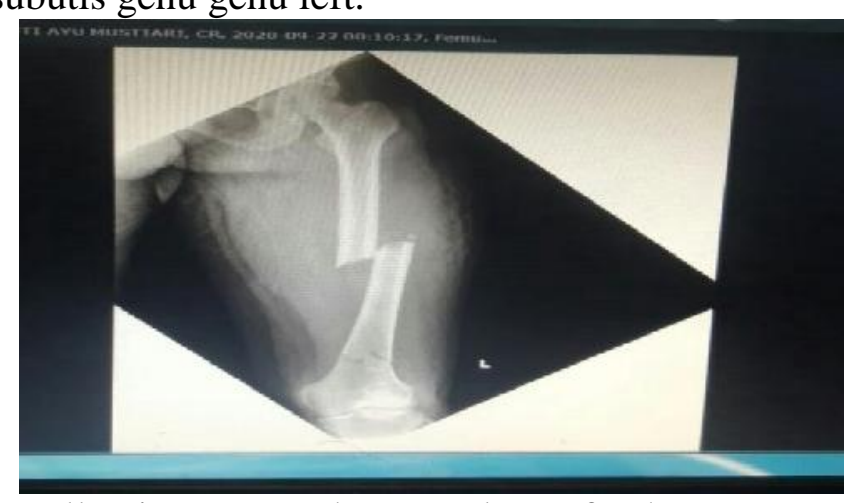

Following are the results of the examination using Roy's adaptation model:

1). Health History

The patient denies a history of hypertension, diabetes mellitus, stroke, asthma and others. The patient has never had surgery and is currently being treated in a hospital.

2). Family Health History

No history of asthma, hypertension, diabetes mellitus, stroke or others genetic disease.

a. Assesment

1) Oxygenation and Circulation

The patient said that he was not short of breath, did not complain of pain when inhaling or when the head was raised or sitting. The patient said he did not have a long history of coughing. Inspection: normal chest shape, symmetrical chest expansion, respiratory rate $24 x /$ minute, intercostal retraction and absent use of respiratory muscles, absent nasal lobe breathing. Palpation: no

tenderness, tactile fremwoods symmetrical right and left chest. Percussion: resonant in both lung fields. Auscultation: vesicular sounds, no abnormal breath sounds.

Bleeding history can not be evaluated, distal area of traction palpable warm, conjuntiv a was anemis with $\mathrm{Hb} 9.05 \mathrm{~g} / \mathrm{dl}$.

2) Eat and Drink

The patient said there was no nausea and vomiting, no anorexia, no decreased appetite,

no difficulty swallowing. The patient said he ate 1 portion of the diet given in the hospital . Inspection: oral mucous membrane was moist, no lesion. The patient's height $157 \mathrm{~cm}$, weight $54 \mathrm{~kg}$ (BMI $21.95 \mathrm{~kg} / \mathrm{m}^{2}$ ) ideal body weight (51.3 to 54 $\mathrm{kg}$ ). No no allergic food. Nutritio nal status is normal.

3) Elimination

Defecate routine once a day with yellow mushy consistency. Spontaneous urinary, no complaints of pain when urinating, clear yellow urine color.

4) Activity

The results of examining the muscle strength of the tractionattached leg cannot be done. The patient has not been able to meet his needs (bathing, toileting). The fall risk assessment based on the Morse Fall Scale (MFS) is on a scale of 70 high risk of falling. Meanwhile, for rest the patient does not experience complaints.

5) Self Protection

Currently the patient has a history of falls in the last three months, does not have more than one disease, meets the needs of a nurse. 
6) Sensation and Pain

The patient felt pain in his left leg with skin traction attached and obtained $\mathrm{P}$ data: none, Q: continued meneus, R: on left leg $\mathrm{S}$ : 3 pain scale, $\mathrm{T}$ : 15 minutes.

7) Fluid and Electrolyte.

In this patient, $\mathrm{Hb}$ decreased 9.05 g / Dl, pale conjunctiva CRT <3 seconds, trauma to the large bone, namely the femur.

8) Neurological and endocrine function: no problems were found.

9) Adaptation

The patient feels ashamed and anxious that the disease will cause disability due to the patient's condition.

10) Role

The patient is a housewife, the patient is just lying down and unable to carry out the role of mother for her child.

b. Nursing Diagnosis

Nursing diagnosis related to mobilization problems include: acute pain associated with physical injury agents characterized by patients complaining of pain, scale 3 , physical mobility impairments associated with decreased muscle strength characterized by decreased range of motion, risk of peripheral neurovascular dysfunction with risk factors for immobilization, risk of falling with risk factors for a decrease in lower extremity strength.

c. Nursing Intervention

The interventions described below focus on nursing diagnoses related to patient mobilization. Acute pain diagnostic interventions associated with physical injury agents are characterized by the patient complaining of pain, a scale of 3 that can be applied is pain management, giving analgesics so that the goal can be achieved, namely the patient can adapt to reduced pain. Barriers to physical mobility are associated with decreased muscle strength, marked by a decrease in the range of motion of the intervention given exercise therapy for muscle control, positioning with the aim of increased mobility. In overcoming the diagnosis of the risk of peripheral neurovascular dysfunction with risk factors for immobilization, interventions that can be given peripheral sensation management, joint movement training therapy so that neurovascular dysfunction does not occur. The risk of falling is the risk factor of decreasing extreme strength under interventions that can be given environmental management of fall prevention, security surveillance by collecting and analyzing information directed about the patient and the environment to be used in improving and maintaining patient safety so that the goal is achieved, namely the patient does not experience falls.

Of all the interventions performed on the above diagnoses the intervention in increasing muscle strength in patients with lower extremity fractures using traction should be considered. Exercises at extremities that do not use traction will be different from muscle exercises at extremities that do not use traction. In fact, patients with impaired mobility must continue to train muscle strength. Interventions to increase muscle strength in the extremities that do not use traction use range of motion exercises while in the extremities that use traction interventions to increase muscle strength is isometric exercises. 


\section{Discussion}

In this case, the management in accordance with the fracture management has been carried out, where the patient has passed the recognize phase, and currently a close reduction using traction is being carried out.

According to Rudolf (2020) management of patients who will undergo open reduction surgery, namely Intermedularry Nail, begins with the installation of traction as another definitive method of management of femoral shaft fractures. In cases of fractures that will not use nail intermedularry, adults rarely use traction because it requires long treatment and the patient is likely to develop infection (Rudloff, 2020).

The traction used in this patient is to use skin traction with the Buck Traction type, namely skin traction where the pull is applied to one plane. This traction is used for comfort before surgery. The use of Buck traction in this case is according to the type of traction and its application.

This type of traction is used on the lower limbs which aims to prevent hip and knee contractures, for preoperative immobilization and to reduce muscle spasm (Black, J. M., \& Hawks, 2014).

The diagnosis that appears when the patient has traction is in accordance with NANDA (2018), namely the diagnosis of mobility constraints, pain and resistance to comfort. The comfort barrier is not taken because the comfort barrier is associated with the treatment program, where traction is important as the first step for intermedularry nailing surgery (Herdman, H. T., \& Kamitsuru, 2018).

Obstacles to physical mobility are associated with decreased muscle strength, marked by decreased range of motion, intervention given exercise therapy for muscle control, positioning with the aim of increased mobility.

Interventions for muscle control need to be considered in this case because the muscle training in the traction-attached leg will be different from the muscle training in the traction-attached leg. Muscle exercises need to be performed on patients with traction installed, because immediately after taking the traction the client immediately feels weak as a result of muscle weakness.

Muscle weakness in patients fitted with skin traction due to immobilization and gravitational pull. This is in patients who use traction to do exercises to increase muscle strength with this type of isometric exercise. Several studies have suggested that isometric exercise has a positive impact on muscle strength.

Dynamic muscle movement refers to isometric exercises where the muscles move short during concentric contraction and lengthen during eccentric contraction (Rittharomya, Aree-ue, Malathum, Orathai, \& Belza, 2020).

Isometric training is an exercise with a static form of exercise that contracts muscles and produces resistance without changing muscle length and without joint movement (Mahmoud, Elnaggar, \& Ahmed, 2017). Isometric exercises are one of the basic therapeutic muscle training types of 3 types of muscle training, namely isometric, isochenetic, isotonic exercises and isometric exercises, which are probably the most appropriate and easily understood by patients and are carried out easily and safely because they don't require special equipment (Anwer \& Alghadir, 2014).

The goal of isometric exercises is first to relieve pain, restore muscle strength. This is in accordance with research conducted by Khosrojerdi in postoperative cases of lower extremity (Khosrojerdi, Tajabadi, Amadani, Akrami, \& Tadayonfar, 2018).

Muscle strength can also be increased through isometric exercises with quardicep exercises such as a study conducted by Rittharomy et al., Where a study was conducted on arthritis patients who were waiting for Total Knee Arthritis action for more than 3 months 
in Thailand (Rittharomya et al., 2020). Isometric exercises with quadrieps on the quadriceps in cases of osteoarthritis can also increase the strength of the quadriceps muscles (Anwer \& Alghadir, 2014). 12 weeks of isometric exercises can also improve muscle strength in patients with obesity and knee arthritis (Mahmoud et al., 2017).

The second objective is to prevent and minimize muscle atrophy when joint movement is not possible due to external immobobilization (Casts, Splints, Traction), activating muscles to initiate restoration of neuromascular control while preserving the healing tissue, increasing postural and joint stability.

Isometric exercises have advantages and disadvantages. The advantage is seen in patients with a static position, the patient has less injury compared to other exercises, requires minimal time, can be done anywhere as long as there is sufficient space, and does not require equipment (Mahmoud et al., 2017). The disadvantage of this exercise is that the strength is formed only in the muscles being trained.

The principles of isomeric training are: 1) The intensity of the exercise that can be generated during isometric muscle contraction. The resulting muscle strength exceeds $60-80 \%$ maximum voluntary contraction (MVC) is successful. 2) The duration of the activity to get adaptive changes in static muscle performance, muscle contraction must be balanced with pause time. This is done to avoid injury due to muscle fatigue.

The effectiveness of isometric training on the increase in muscle strength before and after exercise was obtained from studies, namely 1) In a study conducted by Khosrojerdi (2018) which stated that there was an increase in good muscle by $53 \%$ in the intervention group while in the control group there was a moderate increase in muscle $63 \%$. In Rittharomya's (2020) study, there was an increase in the quardricep exercise muscle, a significant increase from the exercise performed in patients with osteoarthritis. in the intervention group compared to the control group.

From the results of the study, it was found that at the right extremity that was carried out the intervention obtained an increase in the average muscle strength from the measurement results with the QMS (quardriep muscle strengh) scale starting from week 2 to week 12, from 118.08 to 127.79 . In the control group, the results of the increase in the average muscle strength were from 119.73 to 120.09. Whereas in the left extremity muscle strength in the intervention group the average increase in muscle strength from week 2 to week 12 was from 121.28 to 131.17. In the control group the average increase in muscle strength from 120.18 to 120.27 .

Research conducted by Anwer (2014) showed an increase in the average muscle strength in osteoarthritis cases from 8.93 to 11.93 in the intervention group. Whereas in the control group it was 9.28 to 9.32 . 4).

Research conducted by Mehmud et al (2017 found an increase in muscle strength in cases of obesity and knee arthritis in the intervention group muscle increase from 148.4 to 175.6 , while the control group 145.7 to 155.9 .

Contraindications to isometric exercises are diseases of the heart and blood vessels. Exercises performed on patients with heart and blood vessel disorders can aggravate the disease. The following is a table of some of the studies mentioned above.

\section{Conclusion}

Based on the results and discussion, it is explained that the effectiveness of isometric muscles on increasing muscle strength in patients with fractures with skin traction attached, it can be concluded that: 1) Patients with impaired physical mobility need to pay attention to the interventions used to increase muscle 
strength when skin traction is attached, 2) Isometric movements increase muscle strength in lower extremity fractures, 3) Quardricep exercise increases quadriceps muscle strength in preoperative patients with skin traction attached, 4) Quardriscep activation which is part of isometric exercise which aims to increase quadriceps muscle strength in carrying out it needs to pay attention to the influencing factors.

\section{References}

Amponsah, G., \& Gorleku, P. N. (2015). Bony Injuries in Trauma Patients Diagnosed by Radiological Examination. Ghana Medical Journal, 49(2), 97-101. https://doi.org/10.4314/gmj.v49i2.6

Anwer, S., \& Alghadir, A. (2014). Effect of isometric quadriceps exercise on muscle strength, pain, and function in patients with knee osteoarthritis: A randomized controlled study. Journal of Physical Therapy Science, 26(5), 745-748. https://doi.org/10.1589/jpts.26.745

Black, J. M., \& Hawks, J. H. (2014). Medical Surgical Nursing: Clinical Management For Positive Outcomes. Misoouri: Elsevier Saunders.

Herdman, H. T., \& Kamitsuru, S. (2018). NANDA International Nursing Diagnoses Definitions and Classification 2018-2020 (Eleventh E). Thieme Publishers, 11(3), 55. Retrieved from https://www.mculture.go.th/mculture_th/download/ king9/Glossary_about_HM_King_B humibol_Adulyadej's_Funeral.pdf Khosrojerdi, H., Tajabadi, A., Amadani, M., Akrami, R., \& Tadayonfar, M. (2018). The Effect of Isometric Exercise on Pain Severity and Muscle Strength of Patients with Lower Limb Fractures : A Randomized Clinical Trial Study. 7(1). https://doi.org/10.5812/msnj.68104. Research

LeMone, Burke, \& Bauldoff. (2016). Medical-Surgical Nursing : Critical Thinking Patient Care (5th ed.; A. linda, ed.). Jakarta: ECG.

Mahmoud, Elnaggar, \&, \& Ahmed. (2017). Influence of Isometric Exercise Training on Quadriceps Muscle Architecture and Strength in Obese Subjects with Knee Osteoarthritis. Www.Ijmrhs.Com International Journal of Medical Research \& Health Sciences, 6(3), 1-9. Retrieved from www.ijmrhs.com

Riskesdas, K. (2018). Hasil Utama Riset Kesehata Dasar (RISKESDAS). Journal of Physics A: Mathematical and Theoretical, 44(8), 1-200. https://doi.org/10.1088/17518113/44/8/085201

Rittharomya, J., Aree-ue, S., Malathum, P., Orathai, P., \& Belza, B. (2020). The Effectiveness of Preoperative Quadriceps Exercise and Diet Control Program for Older Adults Waiting for Total Knee Arthroplasty: A Randomized Controlled Trial. (4), 485-502.

Rudloff, M. I. (2020). FRACTURES OF THE LOWER EXTREMITY. In Campbell's Operative Orthopaedics, 4-Volume Set (Thirteenth). https://doi.org/10.1016/B978-0-32337462-0.00054-9

Surahman, Rachmat, \& sudibyo. (2016). Metodologi penelitian. Retrieved from http://library1.nida.ac.th/termpaper6 /sd/2554/19755.pdf

Willmott, H. (2015). Trauma and Orthopaedics at a Glance. Retrieved from http://ebookcentral.proquest.com/lib /indonesiauebooks/detail.action?docID=199875 2 